\title{
Cisplatin triggers cancer stem cell enrichment in platinum-resistant cells through NF-KB-TNFa-PIK3CA loop
}

\author{
Bhushan Thakur ${ }^{1,2}$ and Pritha Ray ${ }^{1,2^{*}}$ (D)
}

\begin{abstract}
Background: Parallel to complex alteration in molecular and cellular events, enrichment of cancer stem cells (CSC) contributes significantly in deliberation and maintenance of cisplatin resistance. Cisplatin mediated CSC enrichment is well established in various cancers, yet the underlying mechanism is largely unknown. Cisplatin also promotes transcriptional upregulation of PIK3CA, hence activating PI3K/AKT signaling in resistant cells. However, such cisplatin-induced transcriptional regulators of PIK3CA and their impact on cancer stem cell population in resistant cells are largely unknown.

Methods: DNA-binding protein pulldown using PIK3CA promoter as bait followed by nLCMS was used to identify, cisplatin-induced potential transcriptional regulators of PIK3CA promoter. PIK3CA promoter activity was estimated by luciferase based reporter assay. ChIP was used to assess interaction of NF-KB with PIK3CA promoter. CSC-enriched side-population was sorted using DCV-dye exclusion methods. All the gene expression levels were assessed using qPCR.

Results: Using a transcription factor pull-down assay with PIK3CA promoter, we identified NF-kB as a prime regulator, which escalates both TNFa and PIK3CA expression only in CSC enriched side-population (SP) but not in non side-population (NSP) in platinum resistant ovarian cancer cells upon cisplatin treatment. This SP-specific NF-kB-TNFa-PIK3CA bi-modal loop, on one hand, maintains persistent activation of NF-kB through TNFa- NF-kB autocrine loop, while NF-KB-PIK3CA loop nurture CSC population under cisplatin treatment. Activation of PI3K/AKT signalling drives SP's into an undifferentiated, anti-apoptotic stage through upregulating P21, P27, CFLIP expression. Contrarily, lack of active NF-KB-TNFaPIK3CA loop makes NSPs vulnerable towards cisplatin and undergoes apoptosis. Altogether, cisplatin enriches cancer stem cells properties in SP fraction, which is evident from increased levels of pluripotency gene OCT4/SOX2/NANOG expression. Disruption of PIK3CA-NF-KB loop by Wortamannin reduces SP fraction by 1.4-1.6 fold in control and treated cells.
\end{abstract}

Conclusion: Together, our study signifies an active role of NF-KB-TNFa-PIK3CA bi-modal loop in cisplatin-mediated promotion and maintenance of CSC-like population in platinum-resistant cells.

Keywords: CSC-enrichment, PIK3CA regulators, NF-KB-TNFa-PIK3CA loop

\section{Background}

Chemotherapy is one of the prevailing methods to manage neoplastic growth. Unfortunately, generation of resistance substantially handicaps the efficacy and results in significant mortality in cancer patients. While molecular alteration in signaling cascades aid in acquirement and maintenance of resistance, a small fraction of inherently resistant cancer

\footnotetext{
*Correspondence: pray@actrec.gov.in

${ }^{1}$ Imaging Cell Signaling and Therapeutics Laboratory, Advanced Centre for Treatment, Research and Education in Cancer (ACTREC), Tata Memorial Centre, Navi Mumbai, India

${ }^{2}$ Homi Bhabha National Institute, Mumbai, Anushakti Nagar, India
}

stem cells (CSC) help in repopulating the chemoresistant tumor [1-3]. In particular, these drug resistant CSC's evolve to resist therapy setbacks resulting in incessant growth and relapse. Hence targeting the CSC component has a great therapeutic potential in therapy-resistant disease. However, theoretically achievable, such objective is extremely challenging and requires in depth understanding of how CSC's response towards chemotherapeutics.

CSC's are a small fraction of heterogeneous tumor population identified by surface markers like CD34+/CD38(AML), ESA+/CD44+/CD24-(low) (breast cancer) and functional properties such as side-population (SP) or 
aldehyde dehydrogenase activity [1]. Though association between CSCs and chemoresistance is well established, the key molecular events involved in the regulation of CSCs remain largely unknown. Till date, differential activation of PI3K/AKT, WNT, NOTCH and NF-kB signaling are linked to maintenance of CSC phenotype and chemoresistance [4-6]. However, the actions and the outcomes of cancer therapeutics on signaling cascades in CSCs still remain poorly understood.

Cisplatin, a DNA damaging agent, also modulates several signalling cues including $\mathrm{C}-\mathrm{ABL}, \mathrm{p} 53$ signaling, MAPK/JNK/ERK, PI3K/AKT, NF- $\mathrm{B}$-signalling, FAK and WNT-signaling [7]. Cisplatin resistance is a net effect of multiple mechanisms that either inhibit apoptosis, promote cell survival, or both. Amongst these, the nuclear Factor-kappa B (NF-kB) has been identified as a key player in platinum resistance [8,9]. A variety of stimuli coalesces on NF- $\mathrm{kB}$ activation, which mediated upregulation of $c F L I P, B C l-X L, X I A P$ and favours survival of cisplatin resistant cells $[8,9]$. NF- $\mathrm{B}$ also prevents cisplatin mediated histone acetylation and BRCA1 nuclear translocation in HNSCC and inhibit cisplatin cytotoxicity [10]. In response to extracellular signals, a number of RTKs can activate NF- $\mathrm{KB}$ via PI3K/AKT or JNK/STAT pathway [11]. In many cancers, hyperactivation of PI3K/AKT, a key survival pathway contributes to tumor growth, metastatic competence, and therapyresistance [12]. Besides RTK mediated activation, PI3K gene is regulated by few central transcription factors (p53, NF-kB, FOXO3a, YB1) in chemosensitive cells [13], however, regulators of PIK3CA in chemoresistant cells particularly in response to drugs has never been identified. We have been investigating the underlying mechanism of upregulated PI3K expression in platinumresistant cells, and demonstrated that in absence of a cisplatin-induced Ser46-phosphorylation, p53 failed to bind and represses PIK3CA promoter leading to activation of PI3K/AKT signalling that actively sustained survival but not proliferation of resistant cells [14]. Though lack of PI3K promoter attenuation by cisplatin in resistant cells is indicative of loss of p53's repressive action, it does not explain the observed increase in PIK3CA expression and points toward a second level of regulation for this critical gene and associated signalling.

In the present study, we identified cisplatin responsive potential transcriptional activators of PIK3CA in resistant cells and explored the consequence of this intricate regulation in preserving resistance and CSC-characteristics. Through DNA-protein pulldown, NF-kB was identified as a key cisplatin responsive transcription factor, which escalated PIK3CA specifically in CSC-enriched SP cells and governed an anti-apoptotic, dormant state. Lack of drug-induced quiescence in non-CSC fraction attributed to their susceptibility towards cisplatin. Cisplatin induced an intricate bi-modal feedback loop between TNF $\alpha-N F-\kappa B$ \& NF-кB-PI3K signalling leading to maintenance of CSC-phenotype. This is probably the first mechanistic report on how cisplatin drives enrichment of CSCs in platinum-resistant cells thereby favouring a tilt towards resistance maintenance.

\section{Methods}

Cell lines and treatment

A2780 (DMEM), A2780-CisR (DMEM), OAW42 (MEM), SKOV3 (RPMI) and TOV21G (RPMI) cells were cultured in the respective media supplemented with $10 \%$ fetal bovine serum (HiMedia) and 1\% penicillin-streptomycin (GIBCO). Treatments of Cisplatin $(10 \mu \mathrm{g} / \mathrm{ml})$, TNF $\alpha(50 \mathrm{ng} / \mathrm{ml})$, Forskolin $(10 \mu \mathrm{M}), \mathrm{LiCl}(40 \mathrm{mM})$ and wortmanin $(50 \mu \mathrm{M})$ were given for $24 \mathrm{~h}$.

\section{DNA-protein pulldown and nLC-MS analysis}

OS4-PIK3CA deletion construct (340 bp) was biotinylated by PCR amplification and DNA-protein pulldown was performed using streptavidin-coupled Dynabeads as described earlier [15]. DNA bound protein fraction was eluted with $0.1 \%$ SDS solution and was digested with trypsin (in-solution method) and subjected to Triple TOF 5500+ nanoscale liquid chromatographic tandem mass spectrometry (nLC-MS/MS) for acquiring peptide spectra.

\section{Data analysis and candidate selection}

Significant protein candidates from each MS/MS spectra were identified using protein pilot software at $0.01 \%$ false discovery rate and $p=0.05$ significance level. From each run, identified proteins were categorised based on GO-'biological' and 'molecular' functioning with GSEA server (http://software.broadinstitute.org/gsea/index.jsp). Only protein signatures associated with transcription factors and associated protein were used for gene family overlap and functional overlap between control and cisplatin treated samples were computed through Venn 2.1.0 server. List of identified proteins is given in Additional file 1: Table S1-S3.

\section{Chromatin immune precipitation (ChIP)}

ChIP was performed as described earlier with few modifications [14]. Briefly, $50 \mu \mathrm{g}$ of crosslinked-chromatin were precipitated with $2 \mu \mathrm{g}$ of NF- $\mathrm{BB}$-specific antibody and DNA was eluted post reverse-crosslinking. ChIP-DNA was analysed with semi-quantitative PCR using whole length PIK3CA promoter primers (primer sequences adapted from [16]). Non-immuno-precipitated chromatin was used as total input control.

\section{Isolation of side population}

Side and non-side population cells are sorted as described earlier [17]. Membrane drug transporter inhibitor 
Verapamil (50 $\mu \mathrm{M}$, Sigma) was used as negative control for gating. Data analysis was performed using DIVA software.

\section{Statistical analysis}

All the quantitative data represents the mean \pm SEM of at least three independent biological experiments and statistical significance were analysed using unpaired Student's t test. $P$ value $\leq 0.05$ was considered as significant. Additional supplementary method and materials (Additional file 8).

\section{Results}

\section{Cisplatin enriched side-population fraction with aug-} mented PIK3CA expression in resistant cells

We had earlier reported increase in CSC-enriched Sidepopulation (SP) during acquirement of cisplatin resistance in A2780 and OAW42 cellular models. This SP fraction was associated with increased spheroid formation, upregulated pluripotent genes, and tumorigenic ability at fewer cell implantation in immunecompromised mice, the well-established characteristic features of CSCs [17]. Hence, to understand the impact of cisplatin on existing CSC-population of chemoresistant cells, we assessed the SP fraction in A2780-CisR cells pre and post cisplatin treatment. Cisplatin enhanced SP fraction from $7.3 \pm 0.7 \%(0.65 \pm 0.45 \%$ with verapamil $)$ to $13.7 \pm 2.6 \%(1.8 \pm 0.6 \%$ with verapamil $)$ (Fig. 1a-c). Similar cisplatin dependent increase in SP fraction was also found in other resistant cell lines like TOV21G (from $5.9 \pm 0.7 \%$ to $10.2 \pm 0.4 \%$ ) and SKOV3 (from $4.9 \pm 0.7 \%$ to $8.4 \pm 1.5 \%$ ) cells (Fig. 1c). These cells showed low level of cell death (15-20\%) upon $24 \mathrm{~h}$ of sub-lethal cisplatin treatment $(10 \mu \mathrm{g} / \mathrm{ml})$ (Additional file 2: Figure S1A). However, drug mediated enrichment of a specific cell fraction (SP) was quite unexpected and therefore we attempted to decipher the molecular mechanism. Recent evidences revealed active involvement of $\mathrm{PI} 3 \mathrm{~K} / \mathrm{AKT} / \mathrm{NF}-\mathrm{kB}$ cue in maintenance and enrichment of colon, breast, and ovarian CSCs [1]. Hence, to understand the impact of PI3K/AKT signalling in CSC, we assessed PIK3CA expression in main population (MP), SP, and non-SP (NSP) fraction of resistant cells pre and post cisplatin treatment. Cisplatin augmented PIK3CA expression in MP (1.4, 1.9 and 1.6 fold) and SP fraction (2.1, 2.2, and 1.7 fold) in A2780-CisR, TOV21G and SKOV3 cells respectively (Fig. 1d, Additional file 2: Figure S1B\&C). However, such increase in PIK3CA expression was not observed in their respective NSP fractions.

\section{DNA-protein pull down identified critical PIK3CA promoter binders in platinum resistant cells}

To identify the potential regulators, we adopted a magnetic bead-separation methodology where DNA-binder proteins were pulled down using biotinylated PIK3CA promoter as a bait. Since pulldown with the full-length PIK3CA promoter $(1 \mathrm{~kb})$ was not feasible due to obvious multi-target complexity, a smaller promoter fragment was used. To select the appropriate fragment of PIK3CA promoter, promoter activity of all four deletion constructs each with p53 binding site were assessed (Fig. 1f). The 5'-PIK3CA deletion construct (OS4-PIK3CA) exhibited maximal promoter activity $(1.5-2.4$ fold) in resistant (A2780-CisR, TOV21G, and SKOV3) cells compared to the full-length promoter, which was further increased $(\sim 1.3$ fold) upon cisplatin treatment (Fig. 1g, Additional file 2: Figure S1D\&E). Bioinformatic analysis with JASPER revealed presence of 116 putative transcription factor-binding sites in OS4, majority of which are also present on the full-length promoter sequence.

Using DNA-protein pull down assay, putative binders were isolated from nuclear extracts of A2780-CisR cells (control or cisplatin treated) and processed for nLC-MS analysis. These MS/MS spectra were analysed by protein pilot/Mascot server and identified 312 and 246 (cisplatin treated) and 863 and 339 (untreated) proteins in two biological replicates of A2780-CisR cells. We clustered protein signatures from each independent replicates of untreated and cisplatin treated A2780-CisR cells based on their 'GO- biological and GO- molecular' functions using GSEA (Fig. 2a-c). Pathway analysis of the identified hits revealed 136 (untreated) and 120 (treated) protein signatures comprised of transcription factors and their associated factors. Protein overlap analysis using VENN 2.1.0 sever revealed 67 common binders (36\% of total hits) in both untreated and cisplatin-treated A2780-CisR cells (Fig. 2d) which comprised of general transcriptional assembly including RNA pol-II, PCNA and EP300 (Fig. 2e). List of these identified proteins is given in Additional file 1: Table S1-3. Apart from these overlapping PIK3CA promoter binders, we found an exclusive set of $69(36.4 \%)$ and $53(27.7 \%)$ hits in untreated and cisplatin treated A2780-CisR cells respectively. These proteins were further clustered based on a) presence in both replicates; b) presence of their interacting partners in replicates and c) predicted binding sites on PIK3CA promoter (Fig. 3a). The top three promoter binders found to be NF- $\mathrm{kB}, \beta$-catenin and CREB which are known to maintain self-renewal and differentiation of CSCs in ovarian (NF-kB) [4], nasopharyngeal ( $\beta$-catenin) [18] and oral cancer (CREB) [19] cells.

\section{Cisplatin mediated induction of NF-KB transcriptionally activated PIK3CA expression in resistant cells}

To compare the specificity of PIK3CA regulation by NF$\kappa \mathrm{B}, \beta$-catenin and $\mathrm{CREB}$, we assessed promoter activity of OS4-PIK3CA in A2780 and A2780-CisR cells after TNF $\alpha$, Lithium chloride $(\mathrm{LiCl})$ and forskolin treatment which are known inducers of these factors. Treatment of 


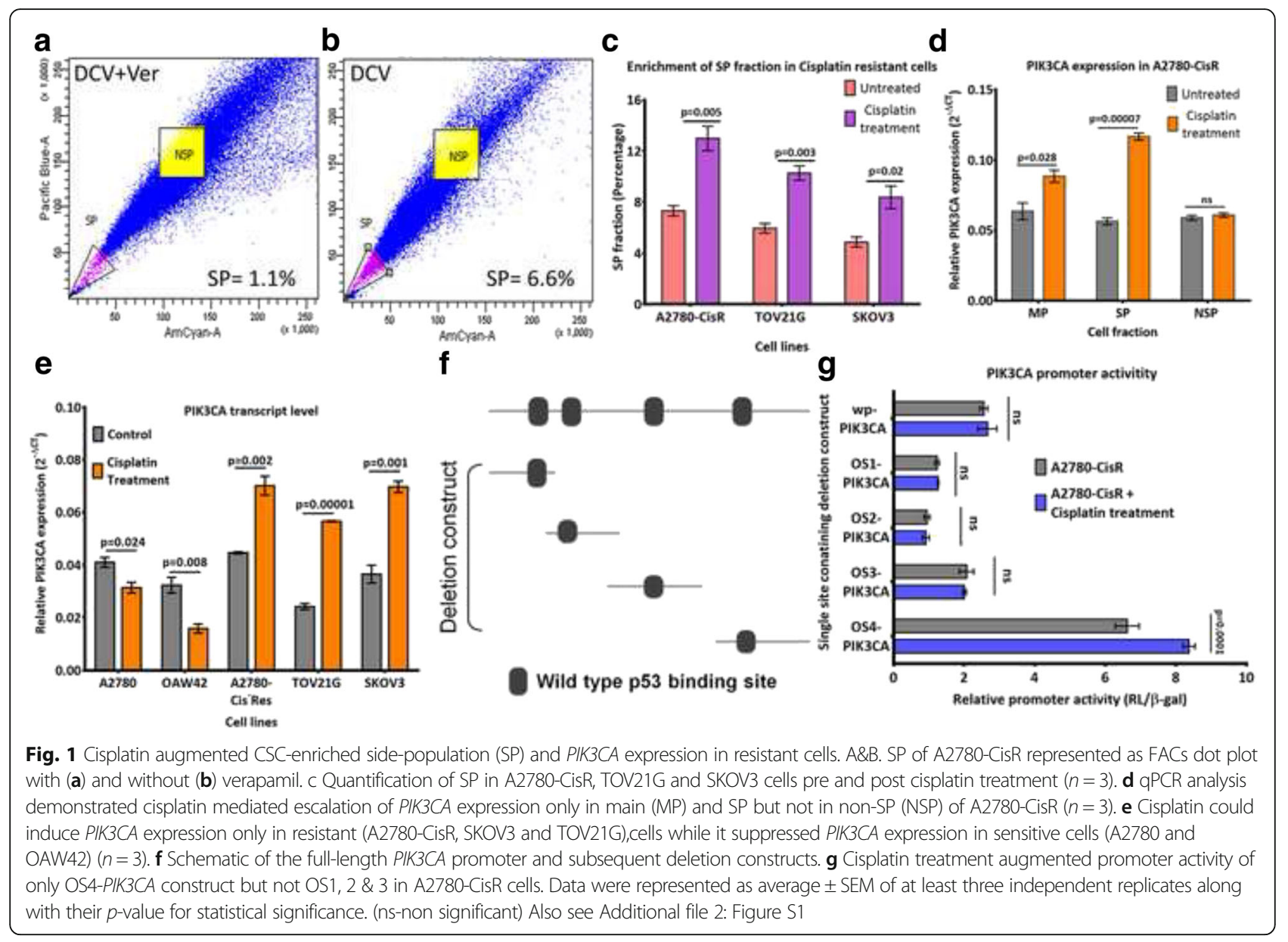

TNF $\alpha$ and $\mathrm{LiCl}$ increased OS4-PIK3CA promoter activity by 2.4 and 1.7 fold in A2780-CisR cells but not in sensitive A2780 cells (Fig. 3b). However, forskolin induced OS4-PIK3CA promoter activity by 1.5 fold in both A2780 and A2780-CisR cells (Fig. 3b) indicating a more general transcriptional regulation. Similar trend was also observed for full-length PIK3CA promoter activity (data not shown). In accordance with promoter activity, TNF $\alpha$ and $\mathrm{LiCl}$ escalated endogenous PIK3CA expression by 2.2 and 1.7 fold in A2780-CisR but not in A2780 cells (Fig. 3c). However, forskolin enhanced PIK3CA expression only in A2780-CisR (1.5 fold) but not in A2780 cells (0.7 fold) (Fig. 3c).

Collectively our data point towards a critical role of $\mathrm{NF}-\mathrm{KB}$ in PI3K/AKT signalling in platinum-resistant cells. Next, to study the SP-specific PIK3CA regulators, we assessed PIK3CA promoter activity in SP fraction after treatment of cisplatin, $\mathrm{TNF} \alpha, \mathrm{LiCl}$ or forskolin. Promoter activity was induced by cisplatin (1.5 fold) and TNF $\alpha$ (1.9 fold) but not by $\mathrm{LiCl}$ (1.1 fold) and forskolin (0.9 fold) (Additional file 3: Figure S2). This was also corroborated with NF-kB binding on PIK3CA promoter, where cisplatin and TNF $\alpha$ enhanced NF-kB occupancy from $1 \%$ to $5 \%$ and $3.8 \%$ of input in A2780-CisR SP fraction. Similarly, TOV21G and SKOV3 cells showed increased NF- $\mathrm{kB}$ binding from $0.3 \%$ to $6 \%$ and $0.1 \%$ to $3 \%$ post TNF $\alpha$ and from $0.3 \%$ to $3.4 \%$ and $0.1 \%$ to $2.7 \%$ post cisplatin respectively (Fig. $3 \mathrm{~d}$ ).

\section{Differential activity of NF-KB was responsible for discrep- ant PIK3CA expression in SP and NSP fraction}

To understand NF- $\mathrm{BB}$ driven PIK3CA promoter regulation, we measured NF- $\mathrm{kB}$ transcriptional activity and PIK3CA promoter activity in MP, SP and NSP fraction of A2780-CisR dual reporter cells [20]. This dual reporter cells expresses a NF- $\mathrm{kB}$ transcriptional reporter (4xNF- $\mathrm{kB}$ binding sites driving a hRL-eGFP) along with the PIK3CA promoter sensor (PIK3CA promoter driving fl2-tdt). SP showed increase in NF- $\mathrm{kB}$ reporter activity after cisplatin (3.5 fold) or TNF $\alpha$ (4.8 fold) treatment (Additional file 4: Figure S3A). Correspondingly, cisplatin or TNF $\alpha$ escalated PIK3CA promoter activity by 1.5 and 1.8 fold in SP cells, respectively (Additional file 4: Figure S3B). Only MP but not NSP showed augmented NF- $k B$ reporter and PIK3CA promoter activity after TNF $\alpha$ treatment. Though cisplatin induced NF- $\mathrm{kB}$ transcriptional 

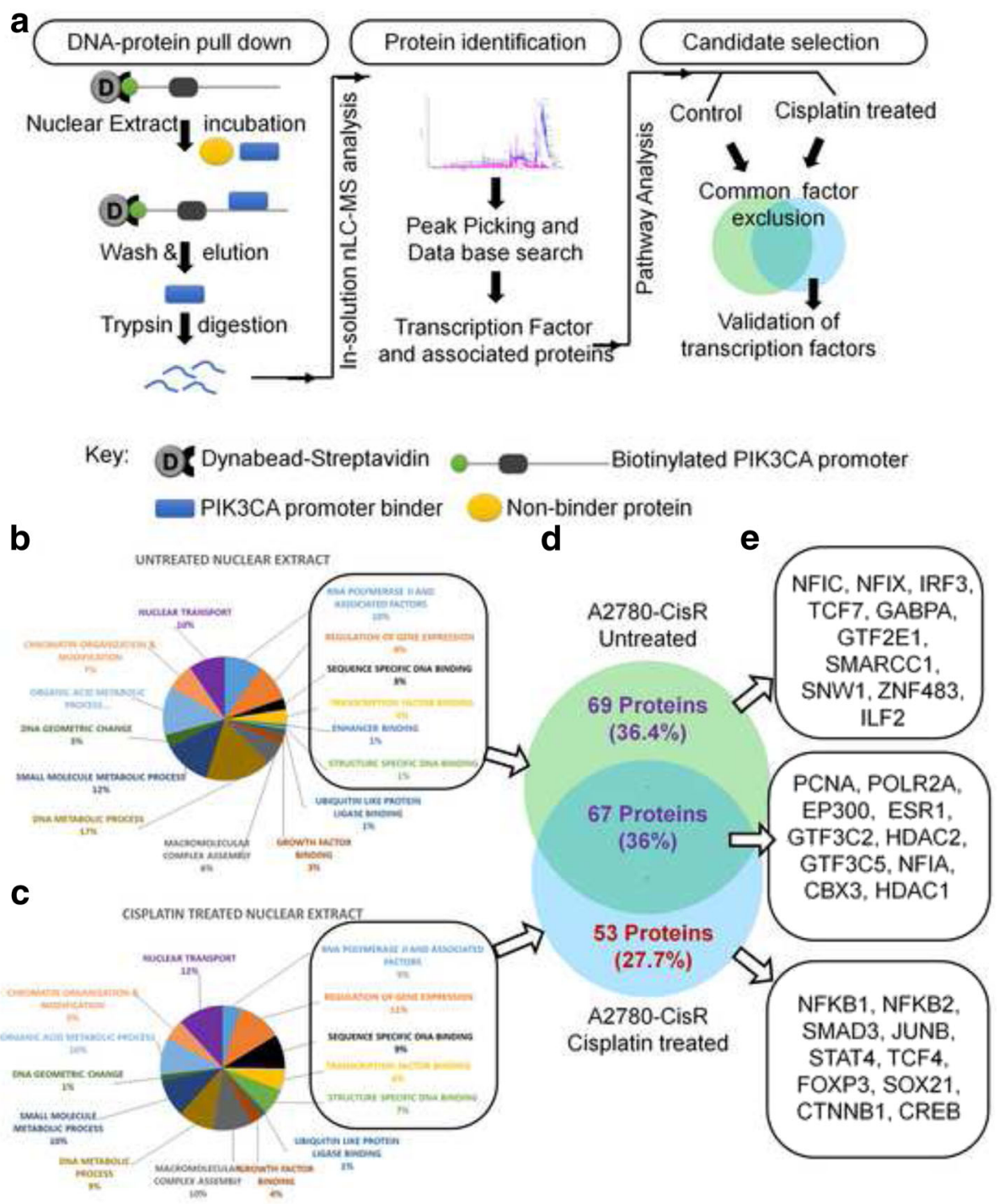

Fig. 2 Identification of cisplatin responsive PIK3CA regulator in resistant cells: a Flow-chart of DNA-protein pulldown assay performed with nuclear extracts of pre and post cisplatin treated A2780-CisR cells. b\&c. Identified protein signatures were functionaly categorised using GSEA server. Only transcription factors and their associated partner proteins were used for further analysis. d\&e. potential binders were grouped from mutually inclusive or exclusive hits from untreated and treated A2780-CisR cells using Venn 2.1.0 server. Ten top scoring factors from each category were listed in the representative boxes. The complete list is given in the Additional file 1: Table S1-S3

activity (2.8 fold), no significant difference was observed in PIK3CA promoter activity in MP cells (Additional file 4: Figure S3). Further, statistical analysis showed a strong positive correlation between NF- $\mathrm{kB}$ reporter activity and PIK3CA promoter activity in SP cells (Pearson correlation, $r=0.96)$. Such correlation was not observed in MP and NSP cells ( $r=0.12$ and $r=0.3$ respectively) (Fig. 3e-g).

Cisplatin treatment induced total NF- $\mathrm{kB}$ level in MP, SP and NSP fraction compared to their untreated counterparts. Notably, control SP showed highest NF- $k B$ level compared to MP and NSP cells (Fig. 3h). Further, NF- $\mathrm{kB}$ binding to PIK3CA promoter in untreated SP cells $(0.9 \%)$, was increased up to 5.7 and $4.3 \%$ post TNF $\alpha$ or cisplatin treatment (Fig. 3i). In accordance with this and to our surprise, enhanced binding of NF-kB upon PIK3CA promoter by TNF $\alpha$ ( $0 \%$ to $2.2 \%$ ) but not by cisplatin $(0 \%$ to $0 \%$ ) was observed in NSP fraction of A2780-CisR cells (Fig. 4g). TOV21G and SKOV3 also showed similar results with physical-interaction of NF-kB with PIK3CA promoter (Additional file 5: Figure S4A-C).

To understand CSC specific NF- $\mathrm{BB}$ activation, we performed co-localization studies for NF- $\mathrm{kB}$ and OCT4 in MP, SP and NSP fraction. Cisplatin treatment to A2780CisR increased nuclear NF- $\mathrm{kB}+$ cells from $5.2 \%$ to $10.8 \%$ (MP) and $17 \%$ to $33.4 \%$ (SP) (Fig. 4a-d). Similarly, TOV21G and SKOV3 cells showed $\sim 2$ fold increase (from 

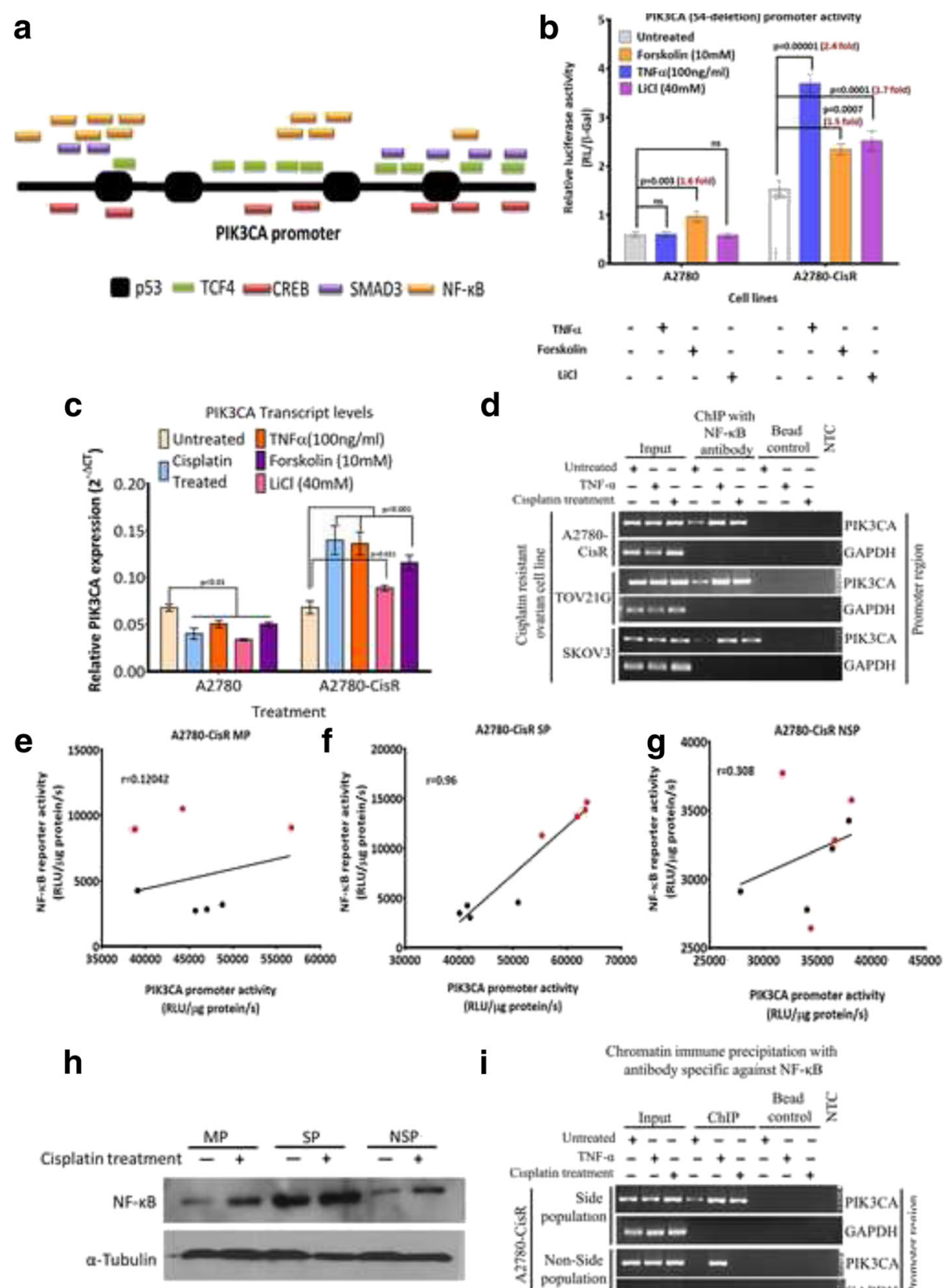

Chromatin immune precipitation with antibody specific against NF-kiB

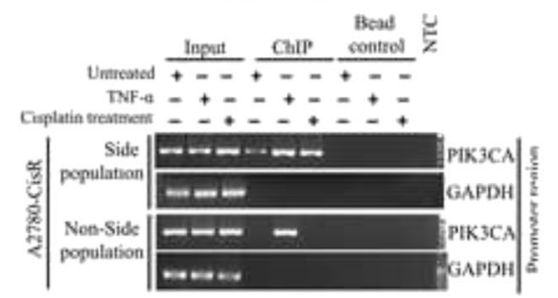

Fig. 3 Cisplatin responsive activation of NF-KB induced PIK3CA in SP fraction of resistant cells. a Schematic of predicted response elements of five transcription factors on full-length PIK3CA promoter identified using JASPER server. $\mathbf{b}$ TNFa and LiCl treatment increased OS4-PIK3CA promoter activity only in A2780-CisR but not in A2780. Forskolin induced marginal change in both cells. Fold change (treated/control) were represented in red font $(n=4)$. c qPCR showed induction in PIK3CA expression only in A2780-CisR but not in A2780 cells after TNFa, LiCl and forskolin treatment. d Both cisplatin and TNFa augmented interaction of NF-KB on PIK3CA promoter as assessed by ChIP assay in A2780-CisR, TOV21G, and SKOV3 cells. e-g Perason correlation analysis showed a strong positive correlation between NF-KB reporter and PIK3CA promoter activity only in SP but not in MP or NSP cells in response to cisplatin. Black -untreated and red -treated. (Note- overlapping values in cisplatin treated MP are appeared as a single dot) $(n=4)$. $\mathbf{h}$ Protein levels of NF-kB pre and post cisplatin treatment. i ChIP assay for NF-kB occupancy on PIK3CA promoter in SP and NSP after TNFa and cisplatin treatment. GAPDH was used as control for ChIP experiments. Data represented as average \pm SEM of four independent replicates with their actual p-value for statistical significance (ns-non significant). Also see Additional file 3: Figure S2, Additional file 4: Figure S3 and Additional file 5: Figure S4

$7.8 \%$ to $14.5 \%(\mathrm{MP})$ and $18.3 \%$ to $27.3 \%(\mathrm{SP})$ in TOV21G, $4.5 \%$ to $8.4 \%$ (MP) and $13.2 \%$ to $21.1 \%$ (SP) in SKOV3) in nuclear NF-kB+ cells (Additional file 6: Figure S5A-D).
Nuclear NF-kB+ cells in NSP fraction remained unchanged after cisplatin treatment in A2780-CisR (3.8\%), TOV21G (5.8\%) and SKOV3 (3.4\%) cells (Fig. 4d, 


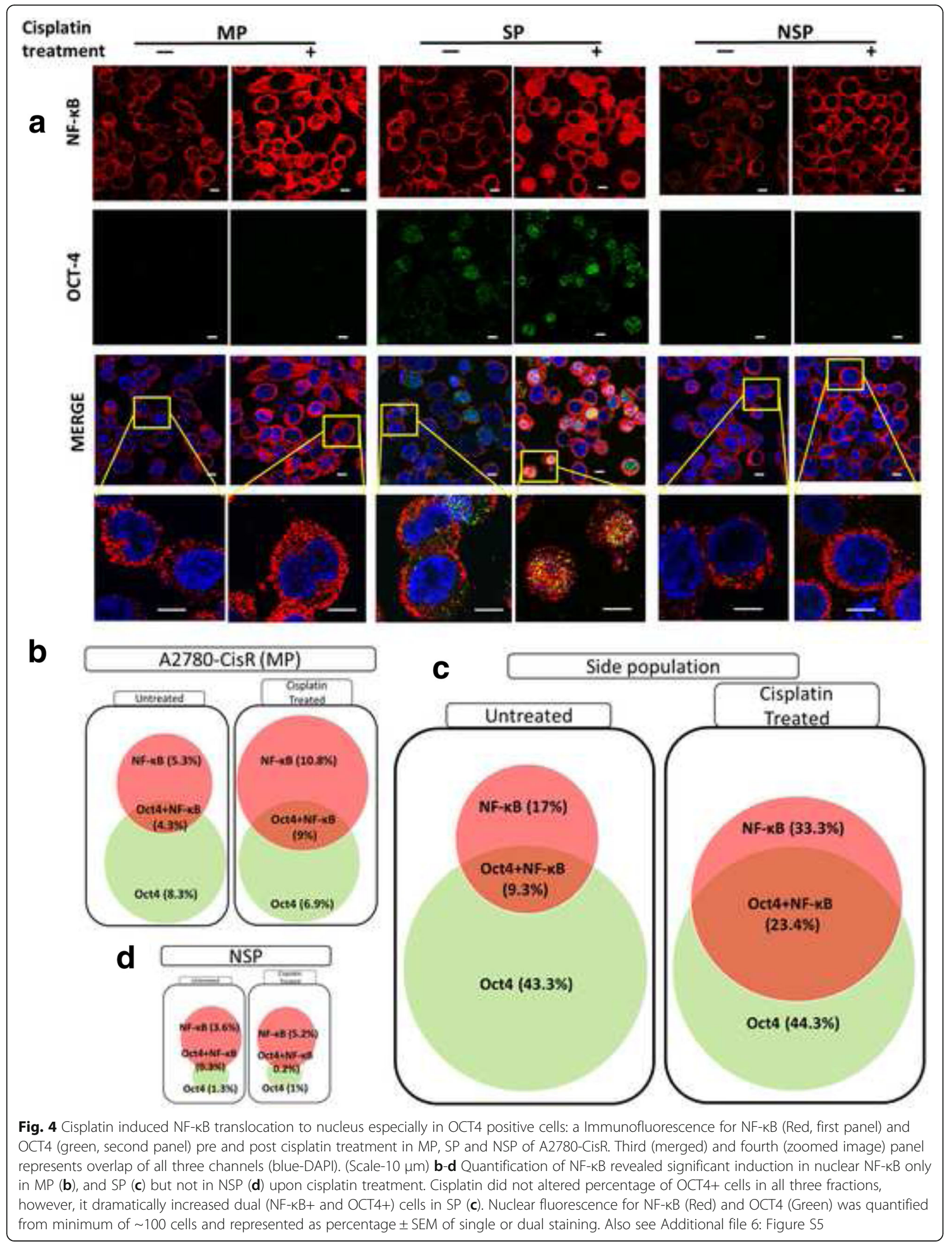


Additional file 6: Figure S5A-D). Further, we quantified nuclear OCT4+ cells to detect CSC fraction from the MP, $\mathrm{SP}$ and NSP fraction. As expected, only SP obtained from A2780-CisR (44.3\%), TOV21G (36.7\%) and SKOV3 (32.4\%) cells showed OCT4+ nuclei and cisplatin treatment did not alter their percent OCT4 positive cells (Fig. 4b-d, Additional file 6: Figure S5E\&F). Notably, SP fraction of A2780-CisR, TOV21G and SKOV3 cells possessed 9.3\%, 10\% and 9\% cells with NF-kB+ and OCT4+ nuclei respectively (Fig. 4, Additional file 6: Figure S5G\&H). Contrarily, MP showed only $4.3 \%, 2.3 \%$, and $1.3 \%$ cells with OCT4+ and NF-kB+ nuclei, while NSP fractions did not show any OCT4+ and NF- $\mathrm{KB}+$ nuclei in A2780-CisR, TOV21G and SKOV3 respectively (Fig. 4b, d, Additional file 6: Figure S5G\&H). These dual positive (OCT4+ and NF-kB+) nuclei were further increased up to $23.4 \%$, $14 \%$ and $15 \%$ post cisplatin treatment in SP obtained from A2780-CisR, TOV21G and SKOV3 cells (Fig. 4c, Additional file 6: Figure S5G\&H). Only MP fraction from A2780-CisR cells demonstrated increase in OCT4+ and $\mathrm{NF}-\mathrm{kB}+$ cells up to $9 \%$ (Fig. 4b), however, such increase was not observed in TOV21G (2.6\%) and SKOV3 (2\%) cells after cisplatin treatment (Additional file 6: Figure S5G\&H). OCT4+ and NF-kB+ cells remained unchanged in NSP of in all three cells (Fig. 4d, Additional file 6: Figure S5G\&H).

\section{Cisplatin triggered enrichment of cancer stem cells in PIK3CA dependent manner}

To understand the impact of PIK3CA expression modulation on the CSC-phenotype, we assessed enrichment of SP fraction in resistant cells post induction of PIK3CA using TNF $\alpha$ or cisplatin. Similar to cisplatin treatment (Fig. 1c), TNF $\alpha$ also augmented SP fraction from $7.3 \pm 0.7 \%$ to 13.6 $\pm 1.9 \%$ in A2780-CisR cells (Fig. 6a, b). In other resistant cells, SP fraction increased from $5.9 \pm 0.7 \%$ to $9.1 \pm 1.6 \%$ (TOV21G) and $4.9 \pm 0.7 \%$ to $7.7 \pm 0.8 \%$ (SKOV3) post TNF $\alpha$ (Additional file 7: Figure S6A\&B). To determine whether cisplatin plays a role in maintenance and/or differentiation of CSC fraction, we repeatedly sorted SP from resistant cells and cultured for three generation as reported earlier [17] (Fig. 5a). We observed that, from $100 \%$ of A2780-CisR sorted SP fraction, only $38.7 \pm 3.5 \%$ of population persisted as SP fraction over $48 \mathrm{~h}$ while rest of the cells differentiated into NSP fraction (Fig. 5b). Interestingly, addition of TNF $\alpha$ or cisplatin in the media led to increase in SP fraction up to $67.1 \pm 7.2 \%$ and $59.9 \pm 12.2 \%$ respectively (Fig. 5b). Similar enrichment in SP fraction was also observed in TOV21G $(31.5 \pm 1.3 \%$ to $45.8 \pm 7.9 \%$ by TNF $\alpha$ and $31.5 \pm 1.3 \%$ to $46.6 \pm 3.9 \%$ by cisplatin) and SKOV3 $(25.1 \pm 3 \%$ to $33.6 \pm 3.1 \%$ by TNF $\alpha$ and $25.1 \pm 3 \%$ to $33.6 \pm 3.1 \%$ by cisplatin) cells (Additional file 7: Figure S6). NSP fraction from all three cells did not show any enrichment in SP fraction with either of the treatments
(Fig. 5b, Additional file 7: Figure S6). To confirm whether this enrichment of SP fraction also enriches CSCcharacteristics, we assessed expression of pluripotency factors (OCT4, NANOG and SOX2) pre and post cisplatin treatment. Cisplatin had also increased one or more pluripotency gene signatures in SP fraction of A2780-CisR (NANOG, SOX2), TOV21G (OCT4), and SKOV3 (OCT4, NANOG) (Fig. 5c-e). MP and NSP fraction did not show such alteration.

\section{Inhibition of PIK3CA activity suppresses cisplatin induced SP enrichment in platinum resistant cells}

We observed relatively higher induction of PIK3CA expression in SP compared to MP and NSP upon cisplatin treatment, which also persuaded enrichment of SP fraction in A2780-CisR, TOV21G and SKOV3 cells. To ascertain that this enrichment depends on PIK3CA activity, we inhibited PI3K-activity using a reversible PI3K inhibitor, wortmannin. PI3K inhibition reduced SP fraction from $7.3 \pm 1.1 \%$ (untreated) to $5.2 \pm 0.6 \%$ (wortmanin) and $14.6 \pm 2.9 \%$ (cisplatin) to $9.1 \pm 0.8 \%$ (cisplatin and wortmannin) in A2780-CisR MP fraction (Fig. 5f). Similar effect was also observed in SKOV3 cells where SP fraction was reduced from $4.8 \pm 1.7 \%$ (untreated) to $1.9 \pm 0.2 \%$ (wortmannin) and $8.5 \pm 1.2 \%$ (cisplatin) to $2.8 \pm 0.4 \%$ (cisplatin and wortmannin) (Fig. 5g). Effect of PI3K inhibition was more robust in serially sorted 3rd generation SP where $\sim 50 \%$ decline in SP-enrichment was evident post wortmannin treatment (24 h). In 3rd generation A2780-CisR cells, PIK3CA inhibition reduced SP from $38.5 \pm 7.1 \%$ (untreated) to $21.6 \pm$ $5.8 \%$ (wortmanin) and $61.3 \pm 9.5 \%$ (cisplatin) to $33 \pm 4.8 \%$ (cisplatin and wortmannin) (Fig. 5f). Similarly SP percentage of 3rd generation SP fraction obtained from SKOV3 cells was reduced from $21.7 \pm 0.4 \%$ (untreated) to $12.3 \pm$ $1.7 \%$ (wortmannin) and $38.3 \pm 4.7 \%$ (cisplatin) to $15.4 \pm$ $0.4 \%$ (cisplatin and wortmannin) (Fig. 5g). NSP cells did not show significant percentage of SP fraction after serial culturing with or without wortmannin treatment (Fig. 5f, g).

To assess the consequence of activated PI3K/AKT cascade on CSCs, we monitored expression of specific downstream targets such as P21, P27, CYCLIN-D1 \& CYCLIN-E1 (cell cycle regulators), BAX, PUMA (pro-apoptotic) and $c F L I P$ (anti-apoptotic) in MP, SP and NSP fractions pre and post cisplatin treatment. Increased P21 and P27 levels and decreased CYCLIN-D1EE1 levels in SP fractions compared to NSP cells marks growth-arrested, quiescent state of SP (Fig. 5h). Interestingly, we observed sharp increase in $C Y C$ LIN-D1 level indicating active proliferation of NSP cells after cisplatin treatment. Though $c F L I P$ levels were increased in all three fractions, transcriptional increment in $B A X$ and PUMA in NSP fractions marked their pro-apoptotic fate (Fig. 5h). However, increased cFLIP levels along with quiescent-state of SP aid these cells to survive the action of chemotherapeutics. In accordance with NF- 


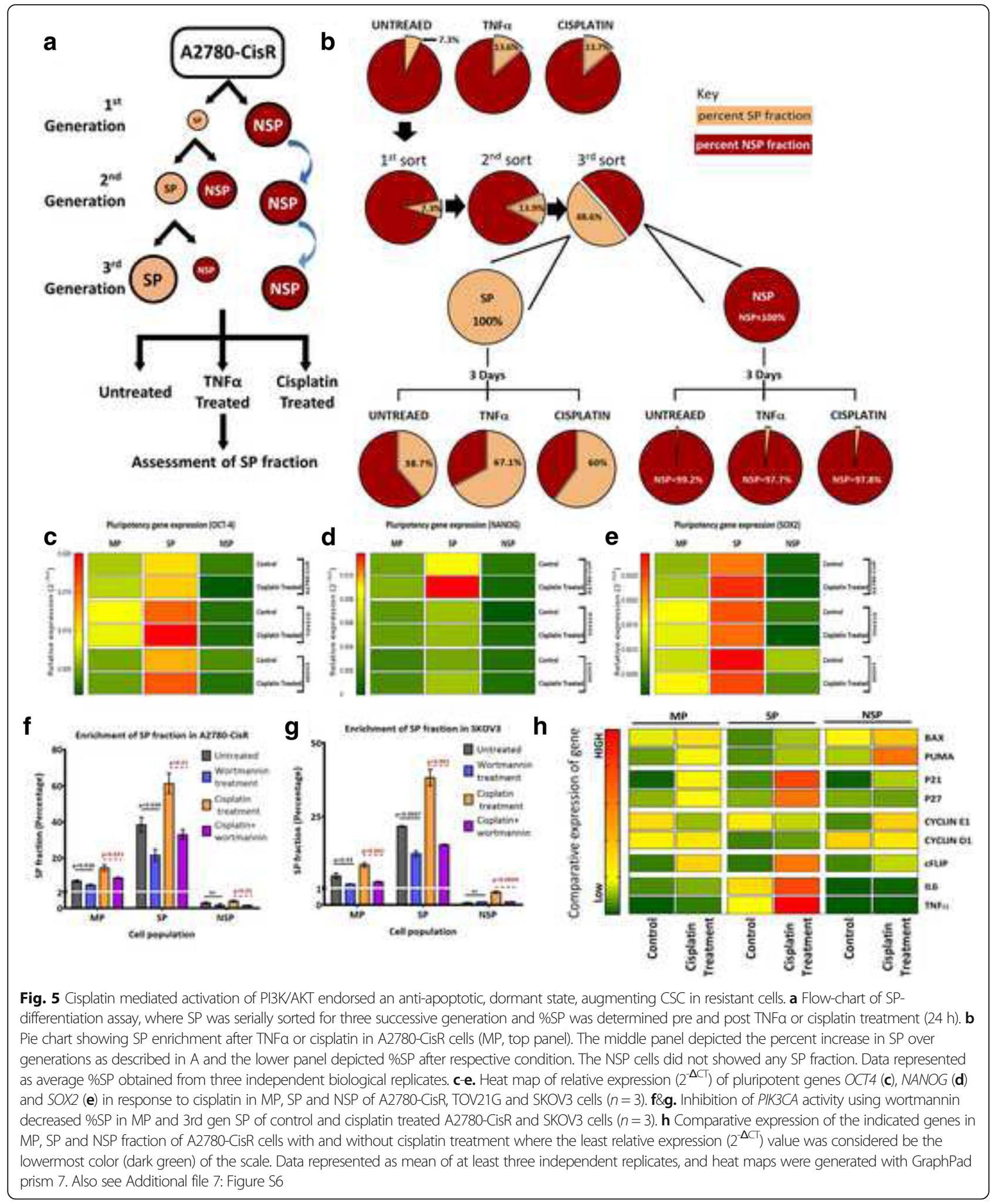

кB-transcriptional reporter activity, its downstream targets (IL6 and TNF $\alpha$ ) also showed higher expression in SP fractions compared to MP and NSP, which further increased significantly upon cisplatin treatment in MP and SP but not in NSP. However, CYCLIN-D1, another target of NF-kB did not show similar modulation in SP fractions. Overall, anti-apoptotic, dormant state provided survival advantage to CSC against 
cisplatin cytotoxicity and led to their enrichment upon cisplatin treatment (Fig. 6).

\section{Discussion}

Chemoresistance, either intrinsic or acquired, substantially handicaps the efficacy of chemotherapy, escalating mortality rates in cancer patients. Acquirement of resistance towards chemotherapeutics is a dynamic and multifactorial process and influenced by cancer stem cell (CSC) enrichment [3, 21, 22]. Though platinum resistance is a common problem for cervical, head and neck, and non-small-cell lung cancer management, it is particularly devastating for epithelial ovarian cancer (EOC) patients as $50 \%$ of the therapy-responders ultimately show chemoresistant-relapse [7, 23]. Utilizing A2780cisplatin resistant cellular model and two naturally occurring cisplatin resistant cells (TOV21G and SKOV3) [14], we attempted to address a unique question of how cisplatin impact existing CSCs in resistant cells. In all these cells, cisplatin enhanced the inherently drug resistant side-population with increased PIK3CA expression. Activation of PI3K/AKT pathway is known to favour cellular endurance against chemotherapeutics and maintenance of CSC-population $[2,18]$ but regulators of this pathway in drug-resistant cells are yet to be identified. Using transcription-factor pulldown assay, we identified NF- $k B, \quad \beta$-catenin and CREB as cisplatin-responsive transcriptional activators of PIK3CA promoter. Amongst them, NF- $\mathrm{BB}$ differentially activated PIK3CA only in CSC-enriched side-population but not in non-SP. Both cisplatin and TNFo induced interaction between NF- $\mathrm{kB}$ and PIK3CA promoter. Further, inhibition of PI3Kactivity dramatically reduced this CSC-enriched SP fraction. Enhanced nuclear co-localization of NF- $\kappa B$ with OCT4 and augmented expression of OCT4, SOX2 and NANOG in SP signified enrichment of CSC properties by cisplatin. Finally, gene expression analysis revealed that cisplatin mediated activation of PI3K/AKT led to an anti-apoptotic, dormant stage, which aided CSC to evade therapeutic action while actively proliferating non-CSC cells succumbed to cisplatin's action. Intriguingly, TNF $\alpha$, a well-known activator of NF- $\mathrm{KB}$ and an NF-kB-regulated gene showed enhanced expression following cisplatin treatment only in SP cells. Collectively, our data emphasize that therapeutic intervention to platinumresistant ovarian cancer cells favours a predominant quiescent state in SP via an interdependent positive feedback loop between TNF $\alpha-N F-k B$ and PI3K/AKT signalling.

Cisplatin forms the first line therapy against malignancies including ovarian, breast and colorectal cancer, however, acquirement of resistance impaired therapeutic efficacy [6, 7]. Amongst several molecular determinants of cisplatin resistance, activated PI3K/AKT pathway turns out to be a key signalling that also aids in survival

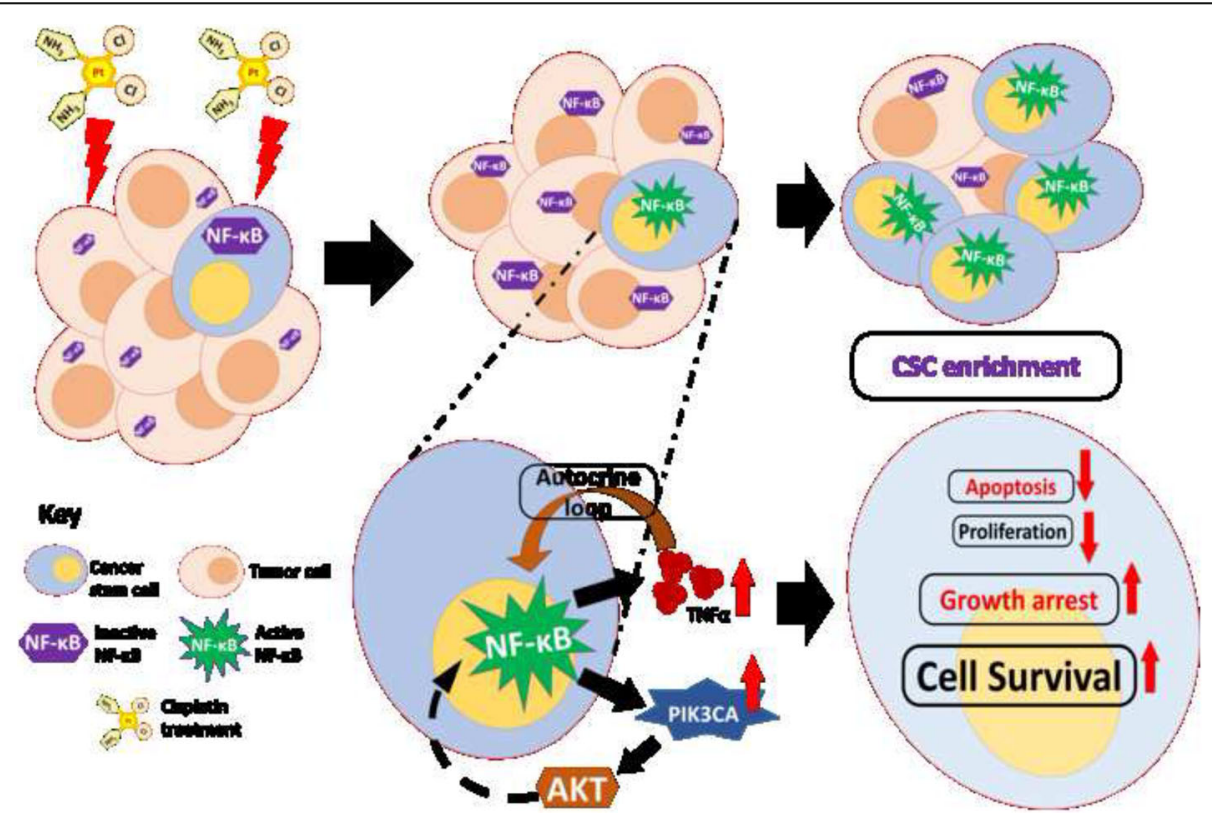

Fig. 6 Proposed model of cisplatin induced CSC enrichment during cisplatin resistance. In platinum resistant cells, cisplatin mediated NF-KB activation only in cancer stem cells, which in turn activates bimodal feedback loop of NF-KB-TNFa and NF-KB-PIK3CA. In one hand, it promotes an autocrine loop by activating TNFa-NF-KB in CSC'C, and on another hand, it increases PIK3CA and PI3K/AKT signalling thus leading to NF-KB stabilisation. Activated PI3K/AKT confers resistance against cisplatin action through modulation of anti-apoptotic (increase in CFLIP) and pro-apoptotic (decrease in BAX and PUMA) genes. A constant supply of NF-KB through TNFa-NF-KB autocrine loop and enhanced stabilisation of NF-KB by activated AKT maintains an anti-apoptotic, quiescent CSC state confers their survival against chemotherapeutics in resistant cells 
of CSCs [1, 2]. Lee et al., (2005) showed that escalated PIK3CA expression in OVCAR-3/CDDP (resistant) cells leads to inhibition of $B A X$ translocation via activated PI3K/AKT conforming platinum-resistance [24]. Amplification of PIK3CA was associated with ovarian and uterine cervical carcinoma, which led to active PI3K/ AKT signaling and resistance acquirement $[25,26]$. However, mechanism for augmented PIK3CA expression is less dissected at molecular level. Intriguingly, we observed cisplatin itself could upregulate PIK3CA expression in resistant but not in sensitive cells [14]. Several transcription-factors (TF) such as p53/p73, cMyc, YB-1, CTF-2, ATF-4, ZNF143, mTFA, AP1, NF$\kappa B$, OCT1, SP1, $\beta$-catenin and CREB are known to be associated with cisplatin resistance, regulating expression of resistance associated genes [27, 28]. Among all these TFs, cisplatin directly influences transcriptional activities of p53, YB-1, ZNF143, mTFA, NF- $к B$, ATF-2, 3 and 4 [27]. We earlier reported that cisplatin-mediated phosphorylation of p53 at Ser46 promotes its binding, and repress PIK3CA promoter in sensitive cells. Absence of such phosphorylation in cisplatin-treated resistant cells resulted in loss PIK3CA attenuation [14] which, however, does not explain the apparent increase in PIK3CA expression level. Till now, only YB-1, NF- $\mathrm{kB}$ and FOXO3a are known to induce PIK3CA expression in unstressed condition [13]. No information is available on regulators of PIK3CA modulation in platinum-resistant cells in response to cisplatin. Thus to identify cisplatinresponsive regulators of PIK $3 C A$ promoter, we subjected PIK3CA promoter bound nuclear fraction (potential transcription-factors) from untreated and cisplatin treated A2780-CisR cells to nLC-MS. We used a short OS4PIK3CA promoter fragment due to: $\mathrm{A}$ ) it shows augmented promoter activity upon cisplatin treatment, B) Contains binding sites for $\mathrm{p} 53$, and other transcription-factors \& C) to avoid multi-target binding complexity possible for fulllength $(1 \mathrm{~kb})$ promoter. Multiple putative candidate proteins bound to PIK3CA promoter in untreated (863 and 339) and cisplatin treated (312 and 246) A2780-CisR cells were identified which were further classified based upon their transcription associated functioning. Among the binders present exclusively in cisplatin treated samples (36\%), we selected the top three PIK3CA binders (NF-kB, $\beta$-catenin and CREB) for further validation. All these three transcription-factors are known to influence cisplatin resistance in various cancer cells. Li et al., (2016) demonstrated that cisplatin-mediated increase in $\beta$-catenin expression aids in resistance-development in oral squamous cell carcinoma [25, 29]. While CREB is shown to be activated by cisplatin in ovarian cancer cells [28] and hyperactivation of NF- $\mathrm{KB}$ is associated with cisplatin resistant human epidermoid carcinoma, KCP-4 cells [30]. Our earlier report also demonstrates that in absence of TLR4/MyD88 signalling, ovarian cancer chemoresistance is maintained by $\mathrm{NF}-\mathrm{kB}$ transcriptional activation under cisplatin treatment [20]. Augmented PIK3CA expression in platinum resistant $\mathrm{H} 460$ cells was also supported with increased NF-kB and $\beta$-catenin but not in CREB levels compared to their parental counterpart [31]. In accordance with this, we observed augmented PIK3CA promoter activity only in platinum resistant cells upon treatment with TNF $\alpha$ (NF-kB inducer) or $\mathrm{LiCl}$ ( $\beta$-catenin inducer), while, forksolin (CREB inducer) increased PIK3CA promoter activity in both sensitive and resistant cells. Though forskolin increased PIK3CA expression in A2780-CisR cells, its treatment to A2780 sensitive cells led to reduction in PIK3CA transcript levels. Other than CREB, forskolin is known to induce binding of Ets-2, phospho-p53 and AP1 TFs to MMP-2 promoter [32]. Hence, observed decrease in PIK3CA expression in A2780 cells may be influenced by other forskolin-induced TFs such as p53. Till date NF-kB's role as a positive regulator for PIK3CA promoter in ovarian cancer cells by TNFo is known [33]. Herein, we demonstrate a unique mechanism of PIK3CA transcriptional upregulation by NF- $\mathrm{kB}$ upon cisplatin, a cytotoxic drug treatment in platinum-resistant cells. ChIP assay showed that NF-kB-PIK3CA promoter interaction increased several fold following TNF $\alpha$ or cisplatin treatment in A2780-CisR, TOV21G, and SKOV3 cells. Our data also suggest $\beta$-catenin complex to be another regulator of PIK3CA promoter, however, presence of only two interacting (SMAD3 \& TCF4) partners of this complex among large numbers of identified binders and minimal ( 1.5) fold increase in PIK3CA expression by $\mathrm{LiCl}$ did not suggest a robust role of this complex. Further experimental studies are required to elucidate the exact contribution of $\beta$-catenin complex.

Two distinct observations that cisplatin mediates SPenrichment and increased NF-kB-PIK3CA promoter interaction, prompted us to assess the expression level of PIK3CA in MP, SP, and NSP fractions. Surprisingly, augmented PIK3CA expression in response to cisplatin found only in SP but not in NSP cells. Next, for comprehensive understanding of what regulates PIK3CA in SP, we treated SP cells with inducers of NF-kB, $\beta$-catenin or CREB. Among them, only TNFa augmented PIK3CA promoter activity in SP but not in NSP cells, indicating NF- $\mathrm{kB}$ as distinct activator of PIK3CA in SP cells. Further, to verify whether SP does contain transcriptionally active NF- $\kappa B$, we used a dual-stable cellular system of A2780-CisR expressing NF-kB-transcriptional reporter and PIK3CA sensor [20]. Cisplatin treatment resulted in strong positive correlation between NF-KB activity and PIK3CA promoter activity only in SP but not in MP or NSP.

Nuclear factor- $\mathrm{kB}$, (NF- $\mathrm{kB}$ ) belongs to a pivotal transcription-factor family, which controls expression of diverse genes related to immune-response, survival, proliferation, angiogenesis, and metastasis. In most cells, 
inhibitor of $\kappa \mathrm{B}$, (I $\mathrm{I} B$ ) regulates NF- $\mathrm{kB}$ transcriptional activity [34] and dissociation of NF- $\mathrm{BB} / \mathrm{I} \kappa \mathrm{B}$ heterodimer leads to nuclear-translocation of NF- $\mathrm{BB}$ where it functions as transcription-factor. The NF- $\mathrm{KB}$ signalling cascade converges with several cellular pathways including PI3K/AKT, where activated AKT promotes IкB degradation via phosphorylating IKK $\alpha$ kinase. In most tumors, NF- $\mathrm{kB}$ signaling is constitutively active, regulating gene induction associated with proliferation (CYCLIN-D1\&D2, CDK2 and c-MYC), growth signals (GM-CSF and IL6), anti-apoptosis (cFLIP, $B C L 2, B c l-x L$ and IAPS) and angiogenesis (VEGF, TNFo, and IL1) [35]. Further, ovarian CD44 + CSCs, survive the treatment of paclitaxel and carboplatin through concomitant activation of NF-kB signalling and conferring resistance against these drugs [4]. Hence, to understand the CSCspecific activation of NF-kB in cisplatin resistant cells, we investigated co-localisation of OCT4 with NF-kB in MP, SP and NSP. 2-3 fold increase in nuclear NF- $\mathrm{kB}+$ and OCT4 + cells in only SP but not in MP or NSP upon cisplatin treatment, clearly indicating contribution of NF- $\mathrm{kB}$ in both CSC-homeostasis and PIK3CA-regulation. Enhanced expression in pluripotency gene (OCT4, SOX2 and $N A N O G)$ by cisplatin exclusively in SP but not in MP or NSP warranted escalation of CSC-characteristics in SP fraction. Interestingly, same drug treatment induced distinct pluripotent gene expression in A2780-CisR, TOV21G, and SKOV3 cells probably due to intercellular differences in genetic constituents. Further to inspect NF- $\mathrm{KB}$ mediated PIK3CA regulation, we assessed NF-kB-PIK3CA promoter interaction in SP and NSP cells pre and post TNF $\alpha$ or cisplatin treatment. Similar to MP, SP but not NSP showed enhanced occupancy of NF-kB on PIK3CA promoter following cisplatin treatment. TNF $\alpha$, however, induced NF$\kappa B$ binding on PIK3CA promoter in both SP and NSP. This seemingly contradictory NF- $\mathrm{kB}-\mathrm{PIK} 3 C A$ promoter interaction by two different stimuli is not surprising as NF- $\mathrm{KB}$ activation by UV-C or doxorubicin is known to induce a complete different set of target genes than that by TNF $\alpha$ and produce entirely different functional consequences [9].

Self-renewal and differentiation are the two major characteristics of stem cells that influence and regulate organogenesis and normal development. Likewise, cancer stem cells control tumor development through self-renewal and differentiation into proliferating tumor cells. If a cytotoxic drug can enrich CSC-population in resistant cells, it would certainly affect one of these two crucial properties. Both cisplatin and TNF $\alpha$ were found to favour a self-renewing undifferentiated state of CSCs as evident from 1.7-2 fold enrichment of SP. In addition, our study revealed that cisplatin mediates CSC-specific activation of NF- $\mathrm{kB}$, which in turn induces expression of TNF $\alpha$ and PIK3CA. This incremented TNF $\alpha$ is known to act as an autocrine cue in concomitant NF- $\mathrm{B}$ activation $[35,36]$, while activation of NF- $\mathrm{kB}$ escalates PIK3CA in CSC's. All these data led us to hypothesize that NF- $\mathrm{KB}$ controlled PIK3CA expression coordinates the CSC-survival and CSC-plasticity under the influence of a chemotherapeutics. Indeed, treating SP with wortamannin, an irreversible PI3K-inhibitor, with and without cisplatin diminished the SP fraction. Gene expression analysis showed increased $P 21, P 27$ and cFLIP and decrease in CYCLIN-D1 and CYCLIN-E1 in cisplatin treated SP cells pointing towards an antiapoptotic, quiescent phase. In contrast, augmented $C Y C$ LIN-DIEE1 expression in cisplatin treated NSP cells marking their proliferative state make them vulnerable towards drug. Activation of PI3K/AKT confers resistance against cisplatin action through up regulation of antiapoptotic genes such as $C F L I P$. Overall, CSC's with active $\mathrm{PI} 3 \mathrm{~K} / \mathrm{AKT}$ and NF- $\mathrm{KB}$ signaling acquire anti-apoptotic, quiescent state conferring survival advantage against action of chemotherapeutic drugs (Fig. 6).

\section{Conclusion}

Platinum-salts are in mainstay of cancer clinic for last few decades and will be continued for decades due to their potent action against proliferating cells. Resistance acquirement against platinum-compounds is a major concern for several malignancies including epithelial ovarian cancer. The unanticipated finding of cisplatin inducing CSC-enriched side-population in platinum-resistant cells opened a new dimension in our understanding on how action of a cytotoxic drug be modulated by cellular ambience. Activated NF- $\mathrm{kB}$ leading to upregulated PIK3CA expression is the key molecular feature behind this action of cisplatin. Though involvement of NF-KB mediated other signalling cascades are possible, our present data signifies PI3K/AKT pathway as the major determinant of cisplatin action in resistant cells. Perturbation in CSC homeostasis through blocking PI3K/ AKT signalling might become a rational approach to intervene Platinum-resistant relapse.

\section{Additional file}

Additional file 1: Table S1. List of protein identified exclusively in A2780CisR control (untreated) cells. Table S2. List of protein identified exclusively in cisplatin treated A2780-CisR cells. Table S3. List of protein identified mutually in untreated and cisplatin treated A2780-CisR cells (XLSX 26 kb)

Additional file 2: Figure S1. Cisplatin augmented PIK3CA expression in TOV21G and SKOV3 cells. A. Cell viability assay with sub lethal treatment of cisplatin $(10 \mu \mathrm{g} / \mathrm{ml})$ for $24 \mathrm{~h}$ revealed $44 \%$ decrease in cell viability in A2780, while resistant cells (A2780-CisR, TOV21G and SKOV3) cells showed 16-24\% reduction in cell viability $(n=4)$. B \& C: Real time quantification of PIK3CA expression revealed treatment of cisplatin augmented level of PIK3CA expression in SP fraction but not in NSP fraction of TOV21G (B) and SKOV3 (C) cells $(n=3)$. D \& E. Only site 4 but not 1, 2 and 3 containing deletion construct showed augmented PIK3CA promoter activity upon cisplatin treatment in TOV21G (D) and SKOV3 (E) cells after transient transfection. All data were represented as average + SEM of at least three independent biological replicates with their actual $p$-value for statistical significance (ns- non significant). (TIFF $360 \mathrm{~kb}$ ) 
Additional file 3: Figure S2. NF-KB escalated PIK3CA promoter activity in SP cells. Cisplatin and TNFa but not lithium chloride or forskolin increased PIK3CA promoter activity in SP collected from A2780-CisR cells ( $n=4$, p-value denoted significant statistical different, ns- non significant). (TIFF $400 \mathrm{~kb}$ )

Additional file 4: Figure S3. NF-KB drove PIK3CA expression in SP cells upon cisplatin treatment. A. Treatment of TNFa or cisplatin dramatically increased renila activity driven by NF-KB response elements only in MP and SP fractions of A2780-CisR dual reporter cell line but not in NSP fraction. TNFa treatment to NSP fraction showed minimal increase in NF-KB transcriptional activity $(n=4)$. B. Similar to NF-KB-reporter activity, TNFa treatment also induced PIK3CA promoter activity in MP, SP and NSP fractions of A2780-CisR dual reporter cell line. However, cisplatin mediated increase in PIK3CA promoter activity was observed only in SP fraction. SP fraction showed much higher induction of PIK3CA promoter activity compared to MP cells after TNFa, or cisplatin $(n=4)$. (TIFF $403 \mathrm{~kb})$

Additional file 5: Figure S4. Cisplatin induced NF-KB and its physical interaction with PIK3CA promoter in TOV21G and SKOV3 cells. A \& B. Similar to A2780-CisR cells, both TOV21G (A) and SKOV3 (B) cells showed higher NF-KB protein levels in SP fraction compared to MP and NSP cells as assessed through immunoblotting. All three fraction from both the cell lines demonstrated cisplatin mediated increase in NF-KB protein levels. C. Similar to A2780-CisR cells, TNFa or cisplatin treatment induced NF-KB occupancy on PIK3CA promoter only in SP fraction of TOV21G and SKOV3 cells. In NSP fraction, only TNFa treatment showed NF-KB binding to PIK3CA promoter in both the cell lines. PCR using primers for GAPDH was used to show purity of chromatin immune-precipitation. (TIFF $357 \mathrm{~kb}$ )

Additional file 6: Figure S5. Cisplatin triggers nuclear localization of NF-KB in OCT4 positive SP cells. A-H. Confocal microscopy for NF-KB and OCT4 staining was performed pre and post cisplatin treatment in TOV21G (upper panel) and SKOV3 (lower panel) cells and quantification were graphically represented. A \& B. Mean nuclear fluorescence intensity measurement demonstrated higher nuclear localization NF-KB in SP fractions of TOV21G (A) and SKOV3 (B) compared to their MP and NSP fractions. Intriguingly, nuclear localization of NF$\mathrm{KB}$ was further increased upon cisplatin treatment in MP and SP fractions of both, TOV21G and SKOV3 cells. C-F. Cisplatin treatment increases only NF-KB nuclear positive cells but not OCT4 positive cells in MP, SP and NSP fractions obtained from TOV21G (C \& E) and SKOV3 (D \& F) cells. G \& H. Similar to A2780CisR, cisplatin increased NF-KB nuclear translocation in SP fractions, especially in OCT4 positive cells of TOV21G and SKOV3 cells. Only MP but not NSP fractions showed nominal increase in co-nuclear localised NF-KB and OCT4 positive cells upon cisplatin treatment. All the quantifications were performed from minimal of $\sim 100$ cells and data was represented as either mean + SEM or percent + SEM. $p$-value represents statistical significance (student t-test) and ns- no significant difference. (TIFF $513 \mathrm{~kb}$ )

Additional file 7: Figure S6. SP differentiation assay for evaluation of CSC enrichment in TOV21G and SKOV3. A \& B. Quantification of percent SP fraction in demonstrated enrichment of SP fraction in MP (top panel) and SP (middle panel) fractions but not in NSP (lower panel) fractions after TNFa or cisplatin treatment compared their untreated counterpart. Represented percentage SP fraction was obtained from three independent biological replicates. (TIFF $240 \mathrm{~kb}$ )

Additional file 8: Supplementary Methods. (DOCX 23 kb)

\section{Abbreviations}

CSC: Cancer stem cells; MP: Main population (whole population); NF-KB: Nuclear factor kappa-light-chain-enhancer of activated B cells; NSP: Non side population (Non-CSC population); PIK3CA: Phosphoinositide-3-kinase catalytic unit alpha; SP: Side population (CSC-enriched population); TNFa: Tumor necrosis factor alpha

\section{Acknowledgements}

Not applicable

\section{Funding}

BT acknowledges CSIR for fellowship. PR acknowledges DST for funding.

\section{Availability of data and materials}

All data generated or analysed during this study are included in this published article [and its Additional files].

\section{Authors' contributions}

BT performed research; BT and PR designed, analyzed research and wrote the paper. All authors read and approved the final manuscript.

Ethics approval and consent to participate

Not applicable

\section{Consent for publication}

Not applicable

\section{Competing interests}

The authors declare that they have no competing interests.

\section{Publisher's Note}

Springer Nature remains neutral with regard to jurisdictional claims in published maps and institutional affiliations.

Received: 19 October 2017 Accepted: 13 November 2017

Published online: 23 November 2017

\section{References}

1. Abdullah LN, Chow EK. Mechanisms of chemoresistance in cancer stem cells. Clin Transl Med. 2013;2(1):3.

2. Kolev VN, Wright QG, Vidal CM, Ring JE, Shapiro IM, Ricono J, Weaver DT, Padval MV, Pachter JA, Xu Q. PI3K/mTOR dual inhibitor VS-5584 preferentially targets cancer stem cells. Cancer Res. 2015;75(2):446-55.

3. Chien J, Kuang R, Landen C, Shridhar V. Platinum-sensitive recurrence in ovarian cancer: the role of tumor microenvironment. Front Oncol. 2013;3:251.

4. Alvero AB, Chen R, Fu HH, Montagna M, Schwartz PE, Rutherford T, Silasi DA, Steffensen KD, Waldstrom M, Visintin I, et al. Molecular phenotyping of human ovarian cancer stem cells unravels the mechanisms for repair and chemoresistance. Cell Cycle. 2009:8(1):158-66.

5. Alison MR, Lin WR, Lim SM, Nicholson LJ. Cancer stem cells: in the line of fire. Cancer Treat Rev. 2012;38(6):589-98.

6. Holohan C, Van Schaeybroeck S, Longley DB, Johnston PG. Cancer drug resistance: an evolving paradigm. Nat Rev Cancer. 2013;13(10):714-26.

7. Wang D, Lippard SJ. Cellular processing of platinum anticancer drugs. Nat Rev Drug Discov. 2005;4(4):307-20.

8. Abedini MR, Qiu Q, Yan X, Tsang BK. Possible role of FLICE-like inhibitory protein (FLIP) in chemoresistant ovarian cancer cells in vitro. Oncogene. 2004;23(42):6997-7004

9. Campbell KJ, Rocha S, Perkins ND. Active repression of antiapoptotic gene expression by RelA(p65) NF-kappa B. Mol Cell. 2004;13(6):853-65.

10. Almeida LO, Abrahao AC, Rosselli-Murai LK, Giudice FS, Zagni C, Leopoldino AM, Squarize CH, Castilho RM. NFkappaB mediates cisplatin resistance through histone modifications in head and neck squamous cell carcinoma (HNSCC). FEBS Open Bio. 2014;4:96-104.

11. Godwin P, Baird AM, Heavey S, Barr MP, O'Byrne KJ, Gately K. Targeting nuclear factor-kappa B to overcome resistance to chemotherapy. Front Oncol. 2013:3:120

12. Mayer IA, Arteaga CL. The PI3K/AKT pathway as a target for cancer treatment. Annu Rev Med. 2016;67:11-28.

13. Kok K, Geering B, Vanhaesebroeck B. Regulation of phosphoinositide 3-kinase expression in health and disease. Trends Biochem Sci. 2009;34(3):115-27.

14. Thakur B, Ray P. p53 loses grip on PIK3CA expression leading to enhanced cell survival during platinum resistance. Mol Oncol. 2016;10(8):1283-95.

15. Drewett V, Molina H, Millar A, Muller S, von Hesler F, Shaw PE. DNA-bound transcription factor complexes analysed by mass-spectrometry: binding of novel proteins to the human c-fos SRE and related sequences. Nucleic Acids Res. 2001;29(2):479-87.

16. Gaikwad SM, Gunjal L, Junutula AR, Astanehe A, Gambhir SS, Ray P. Non-invasive imaging of Phosphoinositide-3-Kinase-catalytic-subunit-alpha (PIK3CA) promoter modulation in small animal models. PLoS One. 2013;8(2):e55971.

17. Singh RK, Dhadve A, Sakpal A, De A, Ray P. An active IGF-1R-AKT signaling imparts functional heterogeneity in ovarian CSC population. Sci Rep. 2016;6:36612.

18. Ma L, Zhang G, Miao XB, Deng XB, Wu Y, Liu Y, Jin ZR, Li XQ, Liu QZ, Sun $D X$, et al. Cancer stem-like cell properties are regulated by EGFR/AKT/betacatenin signaling and preferentially inhibited by gefitinib in nasopharyngeal carcinoma. FEBS J. 2013;280(9):2027-41.

19. Misuno K, Liu X, Feng S, Hu S. Quantitative proteomic analysis of sphere-forming stem-like oral cancer cells. Stem Cell Res Ther. 2013;4(6):156. 
20. Gaikwad SM, Thakur B, Sakpal A, Singh RK, Ray P. Differential activation of NF-kappaB signaling is associated with platinum and taxane resistance in MyD88 deficient epithelial ovarian cancer cells. Int J Biochem Cell Biol. 2015; 61:90-102.

21. Gasch C, Ffrench B, O'Leary JJ, Gallagher MF. Catching moving targets: cancer stem cell hierarchies, therapy-resistance \& considerations for clinical intervention. Mol Cancer. 2017;16(1):43.

22. Shibue T, Weinberg RA. EMT, CSCS, and drug resistance: the mechanistic link and clinical implications. Nat Rev Clin Oncol. 2017;14(10):611-29.

23. Matulonis UA, Sood AK, Fallowfield L, Howitt BE, Sehouli J, Karlan BY. Ovarian cancer. Nat Rev Dis Primers. 2016;2:16061.

24. Lee $\mathrm{S}$, Choi EJ, Jin C, Kim DH. Activation of PI3K/Akt pathway by PTEN reduction and PIK3CA mRNA amplification contributes to cisplatin resistance in an ovarian cancer cell line. Gynecol Oncol. 2005;97(1):26-34.

25. Kolasa IK, Rembiszewska A, Felisiak A, Ziolkowska-Seta I, Murawska M, Moes J, Timorek A, Dansonka-Mieszkowska A, Kupryjanczyk J. PIK3CA amplification associates with resistance to chemotherapy in ovarian cancer patients. Cancer Biol Ther. 2009;8(1):21-6.

26. Bertelsen BI, Steine SJ, Sandvei R, Molven A, Laerum OD. Molecular analysis of the PI3K-AKT pathway in uterine cervical neoplasia: frequent PIK3CA amplification and AKT phosphorylation. Int J Cancer. 2006;118(8):1877-83.

27. Torigoe T, Izumi H, Ishiguchi H, Yoshida Y, Tanabe M, Yoshida T, Igarashi T, Niina I, Wakasugi T, Imaizumi T, et al. Cisplatin resistance and transcription factors. Curr Med Chem Anticancer Agents. 2005;5(1):15-27.

28. Bicaku E, Xiong $Y$, Marchion DC, Chon HS, Stickles XB, Chen N, Judson PL, Hakam A, Gonzalez-Bosquet J, Wenham RM, et al. In vitro analysis of ovarian cancer response to cisplatin, carboplatin, and paclitaxel identifies common pathways that are also associated with overall patient survival. Br J Cancer. 2012;106(12):1967-75.

29. Li L, Liu HC, Wang C, Liu X, Hu FC, Xie N, Lu L, Chen X, Huang HZ Overexpression of beta-catenin induces Cisplatin resistance in oral Squamous cell carcinoma. Biomed Res Int. 2016;2016:5378567.

30. Oiso S, Ikeda R, Nakamura K, Takeda Y, Akiyama S, Kariyazono H. Involvement of NF-kappaB activation in the cisplatin resistance of human epidermoid carcinoma KCP-4 cells. Oncol Rep. 2012;28(1):27-32.

31. Sun Y, Zheng S, Torossian A, Speirs CK, Schleicher S, Giacalone NJ, Carbone DP, Zhao Z, Lu B. Role of insulin-like growth factor-1 signaling pathway in cisplatinresistant lung cancer cells. Int J Radiat Oncol Biol Phys. 2012;82(3):e563-72.

32. Staun-Ram E, Goldman S, Shalev E. Ets-2 and p53 mediate cAMP-induced MMP-2 expression, activity and trophoblast invasion. Reprod Biol Endocrinol. 2009;7:135.

33. Yang N, Huang J, Greshock J, Liang S, Barchetti A, Hasegawa K, Kim S, Giannakakis A, Li C, O'Brien-Jenkins A, et al. Transcriptional regulation of PIK3CA oncogene by NF-kappaB in ovarian cancer microenvironment. PLoS One. 2008;3(3):e1758.

34. Viatour P, Merville MP, Bours V, Chariot A. Phosphorylation of NF-kappaB and IkappaB proteins: implications in cancer and inflammation. Trends Biochem Sci. 2005;30(1):43-52

35. Hoesel B, Schmid JA. The complexity of NF-kappaB signaling in inflammation and cancer. Mol Cancer. 2013;12:86.

36. Zhou A, Scoggin S, Gaynor RB, Williams NS. Identification of NF-kappa B-regulated genes induced by TNFalpha utilizing expression profiling and RNA interference. Oncogene. 2003;22(13):2054-64.

\section{Submit your next manuscript to BioMed Central and we will help you at every step:}

- We accept pre-submission inquiries

- Our selector tool helps you to find the most relevant journal

- We provide round the clock customer support

- Convenient online submission

- Thorough peer review

- Inclusion in PubMed and all major indexing services

- Maximum visibility for your research

Submit your manuscript at www.biomedcentral.com/submit 